\title{
Decision-making Model of Virtual Power Plant for Participating in Spot Market Transaction Based on Hybrid Stochastic and Robust Approach
}

\author{
Dong Jun, Nie Linpeng*, Pa Lidan \\ School of Economics and Management, North China Electric Power University, Beijing, China \\ Email address: \\ dongjun624@126.com (Dong Jun),nlp1992@sina.cn(Nie Linpeng), 1980989576@qq.com (Pa Lidan) \\ ${ }^{*}$ Corresponding author
}

\section{To cite this article:}

Dong Jun, Nie Linpeng, Pa Lidan. Decision-making Model of Virtual Power Plant for Participating in Spot Market Transaction Based on Hybrid Stochastic and Robust Approach. American Journal of Environmental and Resource Economics. Vol. 4, No. 1, 2019 , pp. $32-43$. doi: 10.11648/j.ajere.20190401.14

Received: March 23, 2019; Accepted: April 26, 2019; Published: May 30, 2019

\begin{abstract}
China is vigorously promoting the reform of the electricity spot market after the notice on the development of pilot projects for the spot electricity market was issued in 2017. At the same time, china is upgrading and renovating its energy structure, in the context of structural reform on the energy supply side, the decentralized form of clean energy utilization will develop rapidly. With the continuous improvement of the trading mechanism in spot market, it has become an inevitable trend that many distributed power resources will be involved in electricity market to participate in market transaction. Therefore, in order to promote distributed energy to participate in spot market, virtual power plant technique is paid increasing attentions. Combining the current hot issue, this paper constructs a decision-making model of virtual power plant for participating in spot market transaction based on hybrid stochastic and robust method, which can provide a quantitative decision analysis tool for virtual power plant operators to participate in spot market transactions. The main contribution of this paper are as follows:1) we proposed a transaction decision model that based on hybrid stochastic optimization and robust optimization methods and example simulation was given to illustrate the effectiveness of the model; 2) this paper focused on the electricity market in china.
\end{abstract}

Keywords: Stochastic Optimization, Robust Optimization, Virtual Power Plant, Transaction Decision-making Model

\section{Introduction}

On August 28, 2017, the General Office of the Development and Reform Commission of the State Council and the Comprehensive Department of the National Energy Administration jointly issued the Notice on Piloting the Construction of the Power Spot Market, which clarified the eight spot pilot areas and stipulated that each market entity should be organized to carry out day-ahead, intraday and real-time electric energy trading, realizing the organic connection between dispatch operation and market trading as well as promoting the safe operation of power system and the effective operation of power market. Besides, in 2018, the Southern Regulatory Bureau of the National Energy Administration issued the "Regulations on the Collection of Power Spot Markets in the Southern provinces (Starting in
Guangdong)" document, which laid the foundation for the simulation operation of the Southern Power Spot Market. These signals also marked the official launch of China's power spot market construction work.

At the same time, with the continuous decline of new energy power generation costs, driven by the smart grid and energy Internet technology as well as the continuous improvement of the power market trading mechanism, many distributed power resources have become an inevitable trend in the power market transactions. In this context, virtual power plants are paid increasing attentions. Virtual power plant (VPP) is an integrated power plant that integrates the energy management system under the control of information, control and communication with small-scale distributed energy resources, and can effectively connect many distributed new energy projects into the power grid and realize their 
participation in market transactions [1]. Yangyang Liu et al proposed hybrid method of stochastic optimization and robust optimization to manage the uncertainties of renewable energy [2]. Congying Wei proposed a bi-level scheduling model for VPP with a large number of distributed resources to reduce the net exchange power deviation [3]. Mahmoud $\mathrm{M}$ et al integrates different developed optimization algorithms based on modification of the big bang big crunch method for virtual power plant realization and the proposed model successfully minimize the purchased energy from the grid. [4].

Pandžić constructed a stochastic model of two-stage mixed integer linear programming with the maximum benefit of VPP as the objective function, and obtained the maximum benefit of VPP in the day-ahead and real-time market [5]. Morteza R integrated wind power plants, energy storage systems and demand response into "price-receiver" virtual power plants, and constructed a decision-making model for virtual power plants in the day-ahead market, real-time market and spinning reserve market based on robust optimization methods and calculated the optimal declaration output in each market through the simulation of the example [6]. Mashhour further explored the joint optimal bidding strategy for virtual power plants in energy market and rotating reserve market, and constructed a nonlinear mixed integer programming model with cross-time constraints [7].

Virtual power plants often contain new energy units such as distributed wind power and photovoltaics. Since these distributed power resources usually have randomness and volatility, it is necessary to consider the uncertainties of wind and solar outputs when studying the optimal scheduling of virtual power plants. The stochastic programming method and the robust optimization method are commonly used in uncertain optimization theory. Dantzig [8] is the first one who introduced the concept of stochastic optimization. In the field of power industry, stochastic programming method is commonly used to select representative discrete scenes by considering the probability distribution of wind-light uncertainty, and select the representative discrete scenes by scene generation and reduction to make optimization decisions [9]. Ying Chen [10] built a two-stage stochastic optimization model for real-time scheduling of distributed power supplies. With the deepening of research, stochastic optimization also shows some defects such as large computation and difficult to generate scene sets [11]. In order to ensure the safe operation of the grid, robust optimization is gradually favored by the academic community due to its safety and stability. Zhang Qianwen [12] used a scheduling mode based on wind power output prediction interval to consider the uncertainty of wind power output, and then established a robust optimal scheduling model. Jiang R [13] used robust optimization to establish a model that minimizes the output cost of thermal power units considering the worst wind power output scenario and hydropower units. However, most of the literatures usually only use random programming methods or robust optimization methods in the process of uncertain parameters, and rarely use the two together. In this way, the advantages of the two methods cannot be fully utilized, which also provides a certain research space for the construction of the decision-making model in this paper.

\section{Theoretical Basis}

\subsection{Stochastic Optimization Method}

Stochastic programming is an operational research branch evolved from linear programming, which can solve mathematical programming problems with objective variables or constraints containing random variables to help people make decision-making problems in uncertain environments. $G$. Dantzig first proposed a two-stage stochastic programming model in the 1950 s to study the random mobility of passengers when setting up flight times at airports [14].

A typical model for linear programming is expressed as:

$$
\left\{\begin{array}{c}
\min C^{T} x \\
A x=B \\
x \geq 0
\end{array}\right.
$$

Among them, A, B, and C are all determined constants, which can represent parameters such as production cost, sales price, total amount of resources, and raw material supply amount. However, these parameters tend to show a certain fluctuation trend due to the influence of uncertain factors such as policy environment and market changes, that is, the determined parameters $\mathrm{A}, \mathrm{B}$, and $\mathrm{C}$ become random variables. Accordingly, the determined linear programming problem becomes an uncertain stochastic programming problem. The fluctuation characteristics of these parameters can be described by a specific probability distribution through mathematically transformation. Obviously, if random variables are introduced in planning problems, the model and optimization results will be more in line with the actual situation, and the decision-making results are more reasonable.

Since the coefficients of the model contain random variables, the solution to the stochastic programming problem becomes complicated. Based on probability theory, the most direct solution to the stochastic programming problem is to replace the random variables in the model with the expected values, transform the stochastic programming model into a general mathematical programming model without random variables, and then solve it with a deterministic mathematical programming algorithm. But Valentin Robu indicated that it is very difficult to get the expected value of a function that has a random variable in it [15]. The uncertainty of these random variables can often be classified into multiple different scenarios, and the number of occurrences of these scenarios can be described by a certain probability distribution.

\subsection{Robust Optimization Method}

This chapter will modify the model using the robust basic principles proposed by Bertsimas, and the basic principles of robust optimization will be explained in this section. In general, the form of the linear optimization model with 
uncertain parameters in a coefficient matrix $\mathrm{A}$ is as follows:

$$
\begin{aligned}
& \min c^{T} x \\
& \text { s.t. } \boldsymbol{A} \boldsymbol{x} \geq \boldsymbol{b} \\
& \boldsymbol{x} \in \boldsymbol{X}
\end{aligned}
$$

When there are also uncertain parameters in the objective function $\mathrm{C}$ and the constraint $\mathrm{b}$, the above equation can be transformed into the following form:

$$
\min \omega
$$

$$
\begin{gathered}
\text { s.t. } w-\boldsymbol{c}^{T} \boldsymbol{x} \geq 0 \\
\boldsymbol{A x}-\boldsymbol{b} y \geq 0 \\
\boldsymbol{x} \in \boldsymbol{X}, y=1
\end{gathered}
$$

After the above transformation, the target coefficient matrix $\mathrm{c}$ as well as the constraint condition $\mathrm{b}$ can be regarded as the coefficient matrix A with uncertain parameters for a robust transformation. Suppose A is a matrix of $m \times n$, and $J_{i}$ is a set of column subscripts $j$ of all uncertain parameters $a_{i j}$ in $i$ row of matrix A. Each uncertain parameter $\mathrm{a}_{\mathrm{ij}}(\mathrm{j} \in J i)$ can be regarded as a random variable that varies within bounded symmetric interval $\left[\bar{a}_{i j}-\hat{a}_{i j}, \bar{a}_{i j}+\hat{a}_{i j}\right]$. Here, $\mathrm{a}_{\mathrm{ij}}$ is uncertain, and its specific change trajectory and trend are unknown. Besides, $\bar{a}_{i j}$ represents the predicted value, and $\widehat{a}_{i j}$ represents the estimated value of the interval change.

Defining the parameter $z_{i j}=\left(a_{i j}-\bar{a}_{i j}\right) / \hat{a}_{i j}$, then $z_{\mathrm{ij}}$ is a symmetrically distributed random variable with values in the interval $[-1,1]$. For each constraint $i$ with uncertain parameters, that is, a robust uncertain budget control coefficient $\Gamma i$ is introduced in the row $\mathrm{i}$ of $A x \geq b$, which values in $[0,|\mathrm{Ji}|]$ (where $|\mathrm{J}|$ represents the number of uncertain parameters contained in the constraint i), and does not have to take an integer. This parameter can be used to adjust the probability level of the solution violation with uncertain parameter constraints. $\Gamma i$ (not greater than the largest integer of $\Gamma \mathrm{i}$ ) uncertain parameters in the $\mathrm{a}_{\mathrm{ij}}$ of the constraint $\mathrm{i}$ can be varied within the interval $\left[\bar{a}_{i j}-\hat{a}_{i j}, \bar{a}_{i j}+\hat{a}_{i j}\right]$.

The remaining coefficients are varied within the interval $\left[\bar{a}_{i j}-\left(\Gamma_{i}-\left\lfloor\Gamma_{i}\right\rfloor\right) \hat{a}_{i j}, \bar{a}_{i j}+\left(\Gamma_{i}-\left\lfloor\Gamma_{i}\right\rfloor\right) \hat{a}_{i j}\right]$, and it is not specified that changed within $\left[\bar{a}_{i j}-\hat{a}_{i j}, \bar{a}_{i j}+\hat{a}_{i j}\right]$. In addition, the uncertain parameter set $\mathrm{U}$ can be defined as:

$$
\begin{gathered}
U=\left\{\left(a_{i j}\right) \mid a_{i j}=\bar{a}_{i j}+z_{i j} \hat{a}_{i j}, \forall i, j \in J_{i}, \forall \mathbf{z} \in \mathbf{Z}\right\} \\
\mathbf{Z}=\left\{\mathbf{z} \| z_{i j}\left|\leq 1, \forall j \in J_{i}, \sum_{j \in J_{i}}\right| z_{i j} \mid \leq \Gamma_{i}\right\}
\end{gathered}
$$

where: $z_{\mathrm{ij}}$ is an element of the vector $Z$.

Therefore, the robust optimization correspondence of the linear programming problem represented by equations (2)-(4):

$$
\begin{gathered}
\min c^{T} x \\
\text { s.t. } \sum_{j=1}^{n} \bar{a}_{i j} x_{j}+\min _{\mathbf{z}_{i} \in \mathbf{Z}_{i}} \sum_{j \in J_{i}} \hat{a}_{i j} x_{j} z_{i j} \geq b_{i} \quad \forall i \\
\boldsymbol{x} \in \boldsymbol{X}
\end{gathered}
$$

Finally, the above formula is further transformed by the dual principle, and the final formula is:

$$
\begin{gathered}
\min c^{T} x \\
\text { s.t. } \sum_{j=1}^{n} \bar{a}_{i j} x_{j}-\Gamma_{i} p_{i}-\sum_{j \in J_{i}} q_{i j} \geq b_{i} \quad \forall i \\
p_{i}+q_{i j} \geq \hat{a}_{i j} y_{j} \quad \forall i, \forall j \in J_{i} \\
-y_{j} \leq x_{j} \leq y_{j} \quad \forall j \\
p_{i}, q_{i j}, y_{i} \geq 0 \quad \forall i, \forall j \in J_{i} \\
\mathbf{x} \in \mathbf{X}
\end{gathered}
$$

In equations (14)-(19), $p_{i}, q_{i j}$ and $y_{j}$ are the supplementary decision variables introduced when the dual transformation is performed. In order to make the probability of violating the constraint $\sum_{j=1}^{n} a_{i,} x_{j} \geq b_{i}$ not to exceed $\varepsilon_{\mathrm{i}}$, the robust control coefficient $\Gamma$ i needs to satisfy:

$$
\Gamma_{i} \geq 1+\Phi^{-1}\left(1-\varepsilon_{i}\right) \sqrt{n_{i}}
$$

where: $\Phi^{-1}$ is the inverse of the cumulative distribution function of the standard normal distribution; $n_{i}$ is the number of uncertain parameters contained in the constraint $i$.

\section{Model Construct}

\subsection{Modeling of Units in Virtual Power Plants}

\subsubsection{Distributed Wind Power}

The output of wind power is random, and its randomness is closely related to wind speed. Although wind speed is an intermittent and uncontrollable variable, a large amount of data indicates that the volatility of wind speed can be expressed by Weibull distribution function, and its probability density function is:

$$
f(\mathrm{v}, \mathrm{k}, \mathrm{o})=\left(\frac{k}{o}\right)\left(\frac{\mathrm{v}}{o}\right)^{k-1} e^{-\frac{v}{\left(-\frac{y^{k}}{o}\right.}}
$$

where $v$ is the wind speed, $k$ is the shape parameter, $o$ is the scale parameter, and the corresponding Weibull probability distribution function can be expressed as: 


$$
P(v)=\int_{0}^{v} f_{v}(v) d v=1-\exp \left(-\left(\frac{v}{o}\right)^{k}\right)
$$

Based on the historical data of local wind speed, the expected value $\mu_{w}$ and standard deviation $\sigma_{w}$ of the wind speed are obtained, and the value of the shape parameter $\mathrm{k}$ and the scale parameter o is obtained by the moment estimation method, that is,

$$
k=\left(\frac{\sigma_{w}^{2}}{\mu_{w}}\right)^{-1.086}, o=\frac{\mu_{w}}{\Gamma(1+1 / k)}
$$

The power generation of wind power varies with the wind speed, and is limited by the relevant technical conditions. When the wind speed is lower than the cut-in wind speed or higher than the cut-off wind speed, the wind turbine will not generate power. However, when the wind speed is between the cut-in and cut-out wind speeds, the output of the wind turbine is determined by the wind speed and generally has the following correspondence:

$$
W= \begin{cases}0, & 0 \leq v<v_{\text {in }} \\ \left(\frac{v^{3}-v_{\text {in }}^{3}}{v_{\text {rated }}^{3}-v_{\text {in }}^{3}}\right) W_{\text {rated }}, & v_{\text {in }} \leq v<v_{\text {out }} \\ 0, & v_{\text {out }} \leq v\end{cases}
$$

\subsubsection{Distributed Photovoltaic}

The randomness of photovoltaic power generation is closely related to the light intensity. Nevertheless, the light intensity is also an intermittent resource with randomness and volatility. According to the research, the intensity of the light radiation obeys the Beta distribution function and the probability density function is as follows:

$$
f(\theta)=\left\{\begin{array}{cc}
\frac{\Gamma\left(\alpha^{p v}\right) \Gamma\left(\beta^{p v}\right)}{\Gamma\left(\alpha^{p v}\right)+\Gamma\left(\beta^{p v}\right)} \theta^{\alpha^{p v}-1}(1-\theta)^{\beta^{p v}-1} & 0 \leq \theta \leq 1, \alpha^{p v} \geq 0, \beta^{p v} \geq 0 \\
0 & \text { others }
\end{array}\right.
$$

Among them, $\theta$ is the light intensity, and $\alpha^{p v}, \beta^{p v}$ are the shape parameters, which can be calculated based on the historical data of the local light radiation. Firstly, the expected value $\mu_{p v}$ and standard deviation $\sigma_{p v}$ of the light intensity are obtained, and then the shape parameters are calculated according to the following formula:

$$
\beta^{p v}=\left(1-\mu_{p v}\right) \times\left[\frac{\mu_{p v} \times\left(1+\mu_{p v}\right)}{\sigma_{p v}{ }^{2}}-1\right], \alpha^{p v}=\frac{\mu_{p v} \beta^{p v}}{1-\mu_{p v}}
$$

Therefore, its probability distribution function can be expressed as:

$$
p(\theta)=\int_{\underline{\theta}}^{\bar{\theta}} f(\theta) d \theta
$$

where $\underline{\theta}$ and $\bar{\theta}$ are the maximum and minimum values of the light intensity respectively.
Finally, the output function of photovoltaic power generation is constructed based on the photoelectric conversion efficiency:

$$
P V_{t}=\theta_{t} S_{P V} r
$$

This paper will calculate the corresponding shape parameters based on historical data of local wind speed and illumination, and construct a probability distribution function with significant statistical distribution characteristics based on Weibull and Beta functions, and then generate a certain number of wind speed and light intensity scenes based on these functions. According to the output function of the wind power and photovoltaic, the corresponding wind power and photovoltaic output scenes are generated, so that the simulated scene is more in line with the local scenery output characteristics and used as the predicted value of the real-time output of the scenery.

\subsubsection{Energy Storage Equipment Modeling}

The modeling nature of energy storage equipment is based on a state transition equation that defines the energy contained in the energy storage system at each time as a function of the charge or discharge power per unit time. The mathematical expression can be expressed as:

$$
E_{t}=E_{t-1}+\left(\eta_{e}^{c} P_{t}^{e s s c}-\frac{1}{\eta_{e}^{d}} P_{t}^{e s s d}\right) \Delta t
$$

Where $P_{t}^{\text {essc }}$ and $P_{t}^{\text {essd }}$ are the charging and discharging powers of the energy storage battery respectively; $\eta_{e}^{c}$ and $\eta_{e}^{d}$ are the charging and discharging efficiency factors, indicating the energy loss associated with the charging and discharging processes, in general, the value of them is less than 1; $\Delta t$ represents the time span. During operation, the capacity of the energy storage device and the charge and discharge power of the energy storage battery have upper and lower bounds, which are expressed as:

$$
\begin{gathered}
\alpha_{t}^{c} P_{t}^{\text {essc,min }} \leq P_{t}^{\text {essc }} \leq \alpha_{t}^{c} P_{t}^{\text {essc,max }} \\
\alpha_{t}^{d} P_{t}^{\text {essd,min }} \leq P_{t}^{\text {essd }} \leq \alpha_{t}^{d} P_{t}^{\text {essd,max }} \\
E_{t}^{\text {min }} \leq E_{t} \leq E_{t}^{\text {max }}
\end{gathered}
$$

Where $P_{t}^{\text {essc, } \max }$ and $P_{t}^{\text {essd, } \max }$ represent the maximum charge and discharge power of the energy storage battery, $P_{t}^{e s s c, m i n}$ and $P_{t}^{\text {essd,min }}$ are respectively expressed as the minimum charge and discharge power of the energy storage battery, $E_{t}^{\min }$ and $E_{t}^{\max }$ respectively represent the minimum and maximum capacity of the energy storage battery, $\alpha_{t}^{c}$ and $\alpha_{t}^{d}$ are the state variables of charge and discharge of the energy storage battery, which are $0-1$ variables. When the energy storage battery is in the charging state, $\alpha_{t}^{c}$ is 1 , otherwise it is 0 ; when the energy storage battery is in the discharging state, $\alpha_{t}^{d}$ is 1 , otherwise it is 0 . 
Besides, the energy storage battery can't be charged and discharged at the same time, so the following constraints should be added:

$$
0 \leq \alpha_{t}^{c}+\alpha_{t}^{d} \leq 1
$$

In addition, the energy storage battery will generate cost in the process of charging and discharging. For the sake of simplicity, the charge and discharge cost is defined as the following linear function:

$$
C_{t}^{e s s}=C^{e s s d} P_{t}^{e s s d}+C^{e s s c} P_{t}^{e s s c}
$$

where $C^{\text {essc }}$ and $C^{\text {essd }}$ are the charge and discharge cost coefficients of the energy storage battery.

\subsubsection{Distributed Gas Generator}

The cost of distributed gas units is mainly divided into two parts, namely operating cost and start-stop cost. The power generation cost function at $\mathrm{t}$ time can be expressed as:

$$
C_{t}^{F}=C_{t}^{O p}+C_{t}^{S u d}
$$

where $C_{t}^{O p}$ is the operating cost and $C_{t}^{S u d}$ is the start- stop cost of the gas unit, which can be linearized as:

$$
\begin{gathered}
C_{t}^{o p}=C^{f} G_{t}^{f} \\
C_{t}^{\text {Sud }}=c^{\text {sus }} \mu_{i t}^{o n}+c^{\text {sud }} \mu_{i t}^{o f f} \\
\mu_{i t}^{o f f}=\mu_{t-1}^{c}-\mu_{t}^{c}+\mu_{i t}^{o n}, \forall \mathrm{t} \in \mathrm{T} \\
\mu_{i t}^{o n}+\mu_{i t}^{o f f} \leq 1 ; \mu_{i t}^{o n}, \mu_{i t}^{o f f} \in\{0,1\}
\end{gathered}
$$

Where $G_{t}^{f}$ is the power generation of the gas unit at time t, $C^{f}$ is the operating cost coefficient of the gas unit, $c^{\text {sus }}$ and $c^{\text {sud }}$ represent the single cost of the start and stop of the gas unit respectively, $\mu_{t}^{c}$ is the operating state of the gas unit, which is 1 when the unit is running and is 0 when it is stopped, $\mu_{i t}^{o n}$ and $\mu_{i t}^{o f f}$ respectively represent the start and stop signals of the gas unit, and they are also the $0-1$ variables. When $\mu_{i t}^{o n}$ is 0 , it means that the unit does not enter the start state at time $t$, and when it is 1 , it means the unit starts at time t. However, when $\mu_{i t}^{o f f}$ is 0 , it means that the unit has not entered the stop state at time $t$, and when it is 1 , it means that the unit enters the stop state at time $t$. After the above conversion, all the start and stop problems of the unit can be converted into linear equation and solved. Finally, the gas unit also needs to meet power constraints and climbing constraints:

$$
\begin{gathered}
\mu_{t}^{c} G^{f \min } \leq G_{t}^{f} \leq \mu_{t}^{c} G^{f \max } \\
-\Delta G^{\max } \leq G_{t}^{f}-G_{t-1}^{f} \leq \Delta G^{\max }
\end{gathered}
$$

\subsubsection{Demand Response}

The virtual power plant aggregated in this paper is "incentive demand response virtual power plant", that is, only the user-side resource is subjected to incentive demand response, and its mathematical model can be expressed as:

$$
\begin{gathered}
0 \leq I B_{t} \leq \eta_{t} L_{t} \\
-\Delta I B^{\max } \leq \Delta I B_{t}-\Delta I B_{t-1} \leq \Delta I B^{\max }
\end{gathered}
$$

Equation (42) represents an incentive type demand response constraint, in which $I B_{t}$ represents the demand response applied to the user at time $t$, that is, cutting off the load, $L_{t}$ is the user load at time $\mathrm{t}$, and $\eta_{t}$ is the ratio of the cutoff load that the user can provide to the user load during the $\mathrm{t}$ period; Equation (43) represents the climbing constraint condition of the incentive type demand response in the adjacent time period, that is, the maximum cut off load power provided by the user in the adjacent time period.

\subsection{Decision-making Model of Virtual Power Plant for Participating in Spot Market}

The robust optimization method is suitable for dealing with the optimization problem with uncertain parameters in the constraint condition. In this problem, the objective function contains the uncertain parameters such as each period electricity price $\lambda_{t}^{D a}$ and $\lambda_{t}^{R t}$ of day-ahead market and the real-time market, so the model needs transformation. According to the robust optimization principle introduced in the previous section, this section is about robust transformation in the model, and the objective function and constraints are:

$$
R_{v p p}=\max \omega
$$

Introduce variables $P_{t}^{D a}, P_{t, s}^{R t^{\prime}}$ and $P_{t, s}^{R t}$ to:

$$
\begin{aligned}
& P_{t}^{D a}=W_{t}^{D a}+P V_{t}^{D a}-L_{t} \\
& P_{t, s}^{R t^{\prime}}=(1-\theta) \Delta_{t, \omega}^{+}-(1+\theta) \Delta_{t, \omega}^{-} \\
& P_{t, s}^{R t}=W_{t, s}+P V_{t, s}+P_{t}^{e s s d, R t}-P_{t}^{e s s c, R t}-P_{t}^{D a}+G_{t}^{f}+I B_{t} \\
& \Delta_{t, s}^{+}=\max \left\{P_{t, s}^{R t}, 0\right\} \\
& \Delta_{t, s}^{-}=\max \left\{-P_{t, s}^{R t}, 0\right\}
\end{aligned}
$$

In the above formula, (45) and (46) are variables introduced to facilitate the robust transformation of the model. Equation (47) represents the purchase/selling power of the virtual power plant in the real-time market, and (48) represents the positive deviation of the real-time market., that is, compared with the excess electricity declared in the day-ahead market, this part of electricity can be sold to the system in the real-time market and earned revenue. Formula (49) indicates the negative deviation of the real-time market, that is, compared with the insufficient electricity declared in the day-ahead market, this part of electricity needs to be purchased from the large grid in the real-time market. For the convenience of solving, (47) and 
(49) needs to be converted into the following linear form:

$$
\Delta_{t, s}^{-} \geq 0
$$

$$
\begin{gathered}
\Delta_{t, s}^{+} \geq 0 \\
\Delta_{t, s}^{+} \geq P_{t, s}^{R t}
\end{gathered}
$$

$$
\Delta_{t, s}^{-} \geq-P_{t, s}^{R t}
$$

Constraints are as follows:

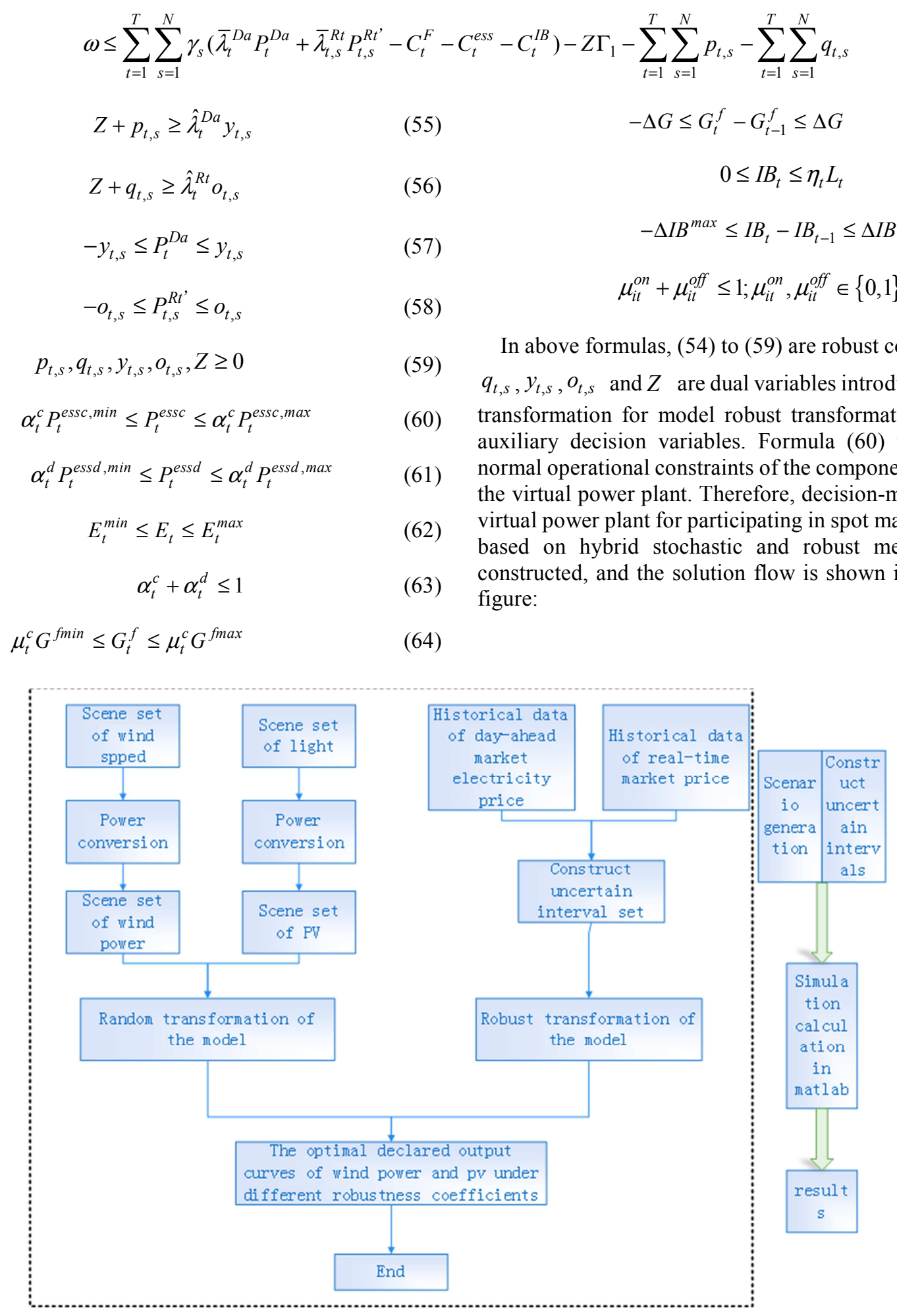

Figure 1. Solution flow based on stochastic robust optimization method. 


\section{Case Analysis}

\subsection{Basic Data}

In order to verify the two-stage stochastic programming decision model constructed in this paper, the distributed wind turbine, the distributed photovoltaic unit and energy storage battery with specific parameters are selected as the example. The rated power of the distributed wind turbine is $8 \mathrm{MW}$, of which the maximum output power is $10 \mathrm{MW}$, and the ramp rate between adjacent period is $3 \mathrm{MW} / \mathrm{h}$. The distributed photovoltaic unit with rated power of $5 \mathrm{MW}$ has a maximum output power of $7 \mathrm{MW}$ and ramp rate is $1.5 \mathrm{MW}$ between adjacent periods. The maximum storage capacity of the energy storage battery is $10 \mathrm{MW} \bullet \mathrm{h}$; the minimum storage capacity is $1 \mathrm{MW} \cdot \mathrm{h}$; the initial capacity is $5 \mathrm{MW} \cdot \mathrm{h}$; the maximum charging power is $1.5 \mathrm{MW} / \mathrm{h}$, and the maximum discharge power is $2 \mathrm{MW} / \mathrm{h}$. In addition, the incentive demand response cost is 500 yuan / MW, the maximum direct control load power does not exceed $20 \%$ of the user load, the gas unit start and stop cost 1000 yuan once, the gas unit rated output power is $5 \mathrm{MW}$, the climbing speed is $1 \mathrm{MW} / \mathrm{h}$, the minimum output power is $1 \mathrm{MW}$, the penalty factor is 0.4 , and the error coefficient of electricity prices between day-ahead market and real-time market is 20\%. Then the CPLEX solver is employed on matlab2016b for calculation.

Table 1. Predictive value of electricity price at each moment in the day-ahead and real-time market.

\begin{tabular}{|c|c|c|c|c|c|}
\hline Trading hours & $\bar{\lambda}_{t}^{D a}[$ yuan/MWh] & $\bar{\lambda}_{t}^{R t}[$ yuan/MWh] & Trading hours & $\bar{\lambda}_{t}^{D a}[$ [yuan/MWh] & $\bar{\lambda}_{t}^{R t}$ [yuan/MWh] \\
\hline 1 & 164.673 & 242.065 & 13 & 168.414 & 188.135 \\
\hline 2 & 137.584 & 317.607 & 14 & 206.26 & 245 \\
\hline 3 & 119.636 & 246.494 & 15 & 302.002 & 316.869 \\
\hline 4 & 108.922 & 159.166 & 16 & 285.103 & 321.223 \\
\hline 5 & 71.478 & 137.362 & 17 & 268.579 & 333.901 \\
\hline 6 & 89.793 & 146.904 & 18 & 441.263 & 521.991 \\
\hline 7 & 68.888 & 118.822 & 19 & 946.712 & 1185.145 \\
\hline 8 & 74.315 & 130.96 & 20 & 904.331 & 1146.879 \\
\hline 9 & 91.807 & 116.235 & 21 & 761.885 & 948.805 \\
\hline 10 & 96.474 & 132.754 & 22 & 632.965 & 639.663 \\
\hline 11 & 136.84 & 155.633 & 23 & 319.958 & 325.248 \\
\hline 12 & 151.5 & 163.32 & 24 & 288.078 & 298.382 \\
\hline
\end{tabular}

\subsection{Analysis of Calculation Results}

\subsubsection{Output Analysis of Virtual Power Plant}

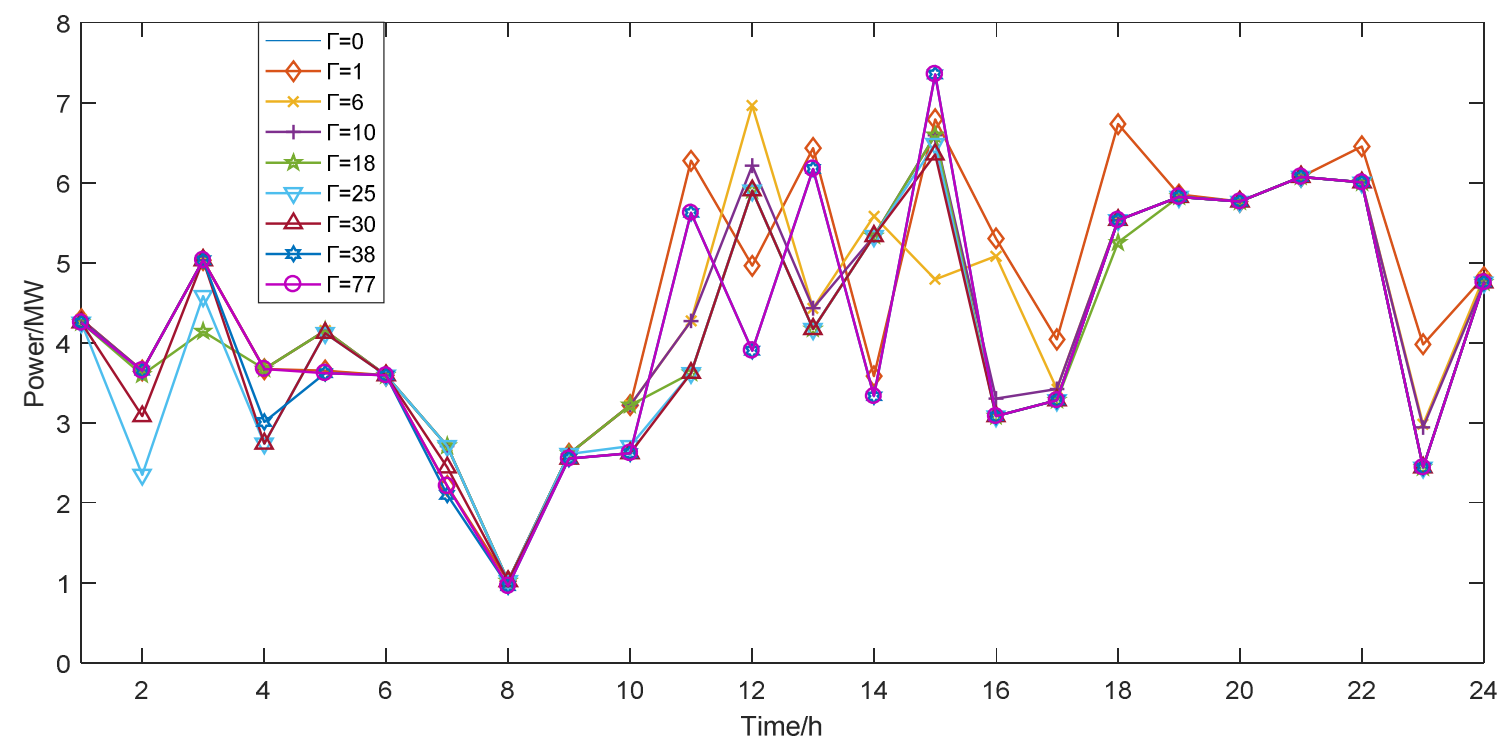

Figure 2. Joint declaration output in day-ahead market of virtual power plant with different scenarios of robustness. 


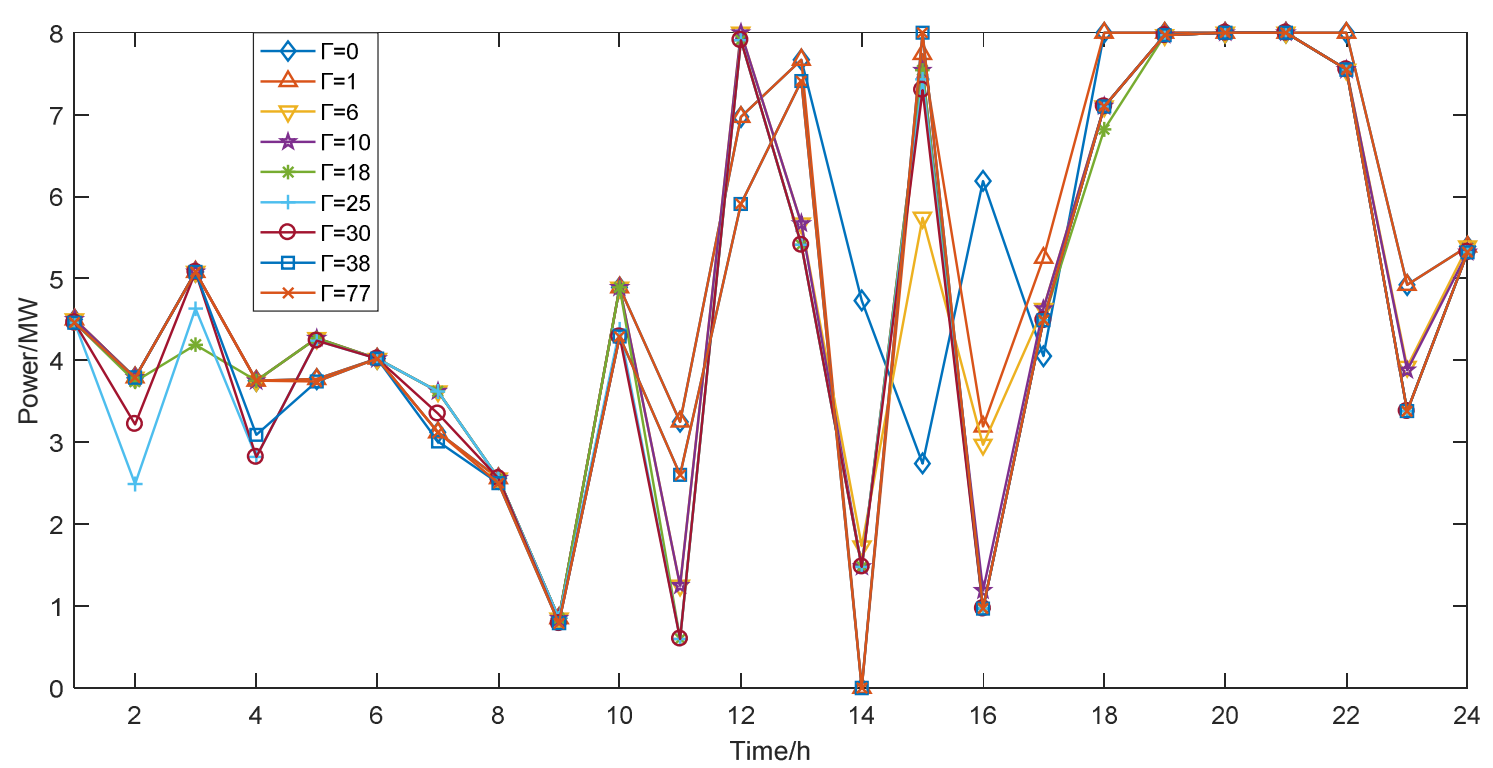

Figure 3. Wind power declaration output in day-ahead market of virtual power plant with different scenarios of robustness.

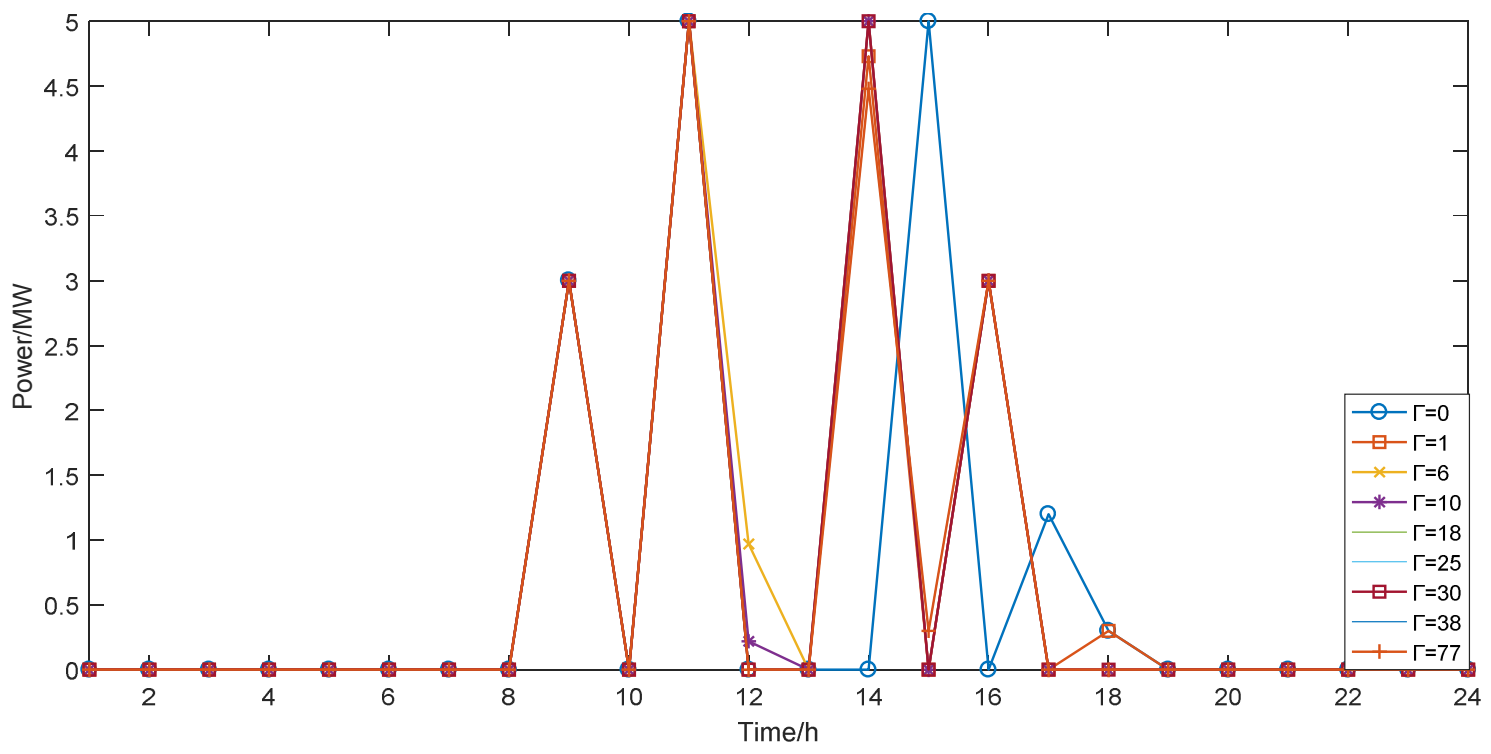

Figure 4. Photovoltaic declaration output in day-ahead market of virtual power plant with different scenarios of robustness.

Figure 3, Figure 4 respectively show the day-ahead market declared output curve of distributed wind power and distributed PV in the virtual power plant, and Figure 2 shows the final day-ahead declaration output plan to the system after meeting user's load demand. It can be seen from Figure 2 to Figure 4 that the values of different robust control coefficients will affect the virtual power plant's day-ahead output plan, and finally contribute to different output curves. In general, the joint power output curves of virtual power plant under different robustness coefficients are relatively stable, and the output times are mainly distributed in three time periods, 0-6 period, 10-16 period and 18-22 period. Besides, the PV output curves are similar. Except when $\Gamma=0$, the PV output curves under the remaining robust coefficients are almost the same, and the concentrated output times are 9, 11,14 , and 16. In contrast, the wind power's declared output curves fluctuate greatly, because wind power itself has large volatility and strong randomness. Therefore, in order to ensure the robustness of the calculation results under the stochastic robust decision model, it is necessary to control the output of wind power. In addition, with the changes of robustness coefficient, the wind power output plan will be adjusted accordingly.

\subsubsection{Benefit Analysis of Virtual Power Plant}

Profit curves with different robustness coefficients and deviation penalty coefficients are shown in Figure 5. The joint profit curves of virtual power plant in day-ahead and real-time markets gradually decrease with the robust uncertain budget coefficient increasing. When the value of $\Gamma$ is between 0 and 18, the profit values fluctuate greatly. Since then, with the value of $\Gamma$ increasing, the profits of the virtual power plant are still decreasing, but the magnitude is gradually slowing down. Decision makers with strong risk 
tolerance can choose the output plan when the value of $\Gamma$ is small. Currently, the profits are higher, but they also need to bear the large risk of violating constraint probability. In addition, with the increase of deviation penalty coefficient, the profits of the virtual power plant are gradually increasing, which indicates that the profits of the virtual power plant in the real-time market are gradually increasing. Although it may affect the profits in day-ahead market, the joint profits in these two markets are still increasing, which also provides a reference of market selection for decision makers in virtual power plants.

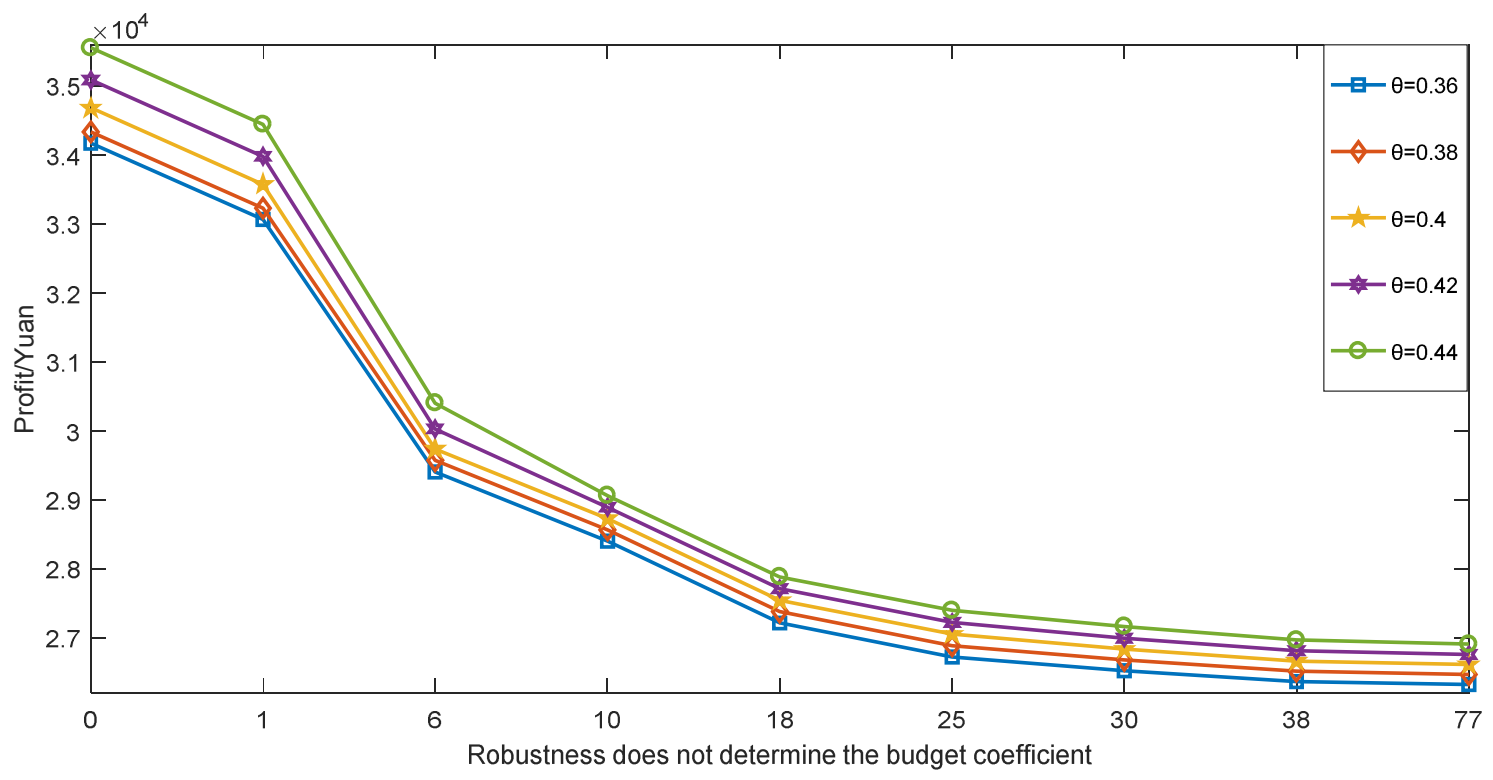

Figure 5. Profit curves with different robustness coefficients and deviation penalty coefficients.

\subsubsection{Performance Comparison Analysis Between Stochastic Robust Optimization and Deterministic Optimization}

Table 2 shows a comparative analysis between different forecasting error intervals of electricity prices in day-ahead and real-time markets. The error means that the predicted values of the various periods in the day-ahead and real-time markets deviate from the actual values by a multiple of the reference curve. With the prediction error coefficient increasing, the cost of real-time operation under the stochastic robust optimization method decreases. This is because the results of the robust optimization method are more conservative. When the market price fluctuates greatly, the decision makers of the virtual power plant hope to report the least possible output plan in the day-ahead decision-making, which will reduce the output of other balance units in the real-time market. The real-time running cost under the stochastic robust optimization method is obviously lower than the deterministic optimization method, but with the operating cost in the real-time phase decreasing, the profit of the virtual power plant is also greatly reduced. However, the deterministic optimization is due to the determination of the market price, of which optimization result does not change with the fluctuation of the market price. Therefore, the deterministic optimization result is fixed value, which is obviously not suitable for the needs of the virtual power plant decision makers.

Table 2. Performance comparisons of optimization methods for different power price error coefficients.

\begin{tabular}{lllllll}
\hline \multirow{2}{*}{ Predicti-on error } & \multicolumn{2}{l}{ Stochastic robust optimization $(\Gamma=6)$} & \multicolumn{3}{l}{ Deterministic optimization } \\
\cline { 2 - 7 } & $\begin{array}{l}\text { Real-time running } \\
\text { cost (yuan) }\end{array}$ & revenue (yuan) & profit (yuan) & $\begin{array}{l}\text { Real-time running } \\
\text { cost (yuan) }\end{array}$ & revenue (yuan) & profit (yuan) \\
\hline $\pm 10 \%$ & 8717.8 & 40889 & 32171.2 & 8717.8 & 43399.75 & 34681.95 \\
$\pm 20 \%$ & 4065.2 & 33802.22 & 29737.02 & 8717.8 & 43399.75 & 34681.95 \\
$\pm 30 \%$ & 2079.8 & 29527.55 & 27447.75 & 8717.8 & 43399.75 & 34681.95 \\
$\pm 40 \%$ & 1985.6 & 27233.34 & 25247.74 & 8717.8 & 43399.75 & 34681.95 \\
\hline
\end{tabular}

Table 3. Performance comparisons of optimization methods for different power price error coefficients.

\begin{tabular}{lcccccccccc}
\hline \multirow{2}{*}{ Time } & \multicolumn{10}{l}{ Performance comparisons of optimization } \\
\cline { 2 - 11 } & $\boldsymbol{\tau}=\mathbf{0}$ & $\boldsymbol{\tau}=\mathbf{1}$ & $\boldsymbol{\tau}=\mathbf{6}$ & $\boldsymbol{\tau}=\mathbf{1 0}$ & $\boldsymbol{\tau}=\mathbf{1 8}$ & $\boldsymbol{\tau}=\mathbf{2 5}$ & $\boldsymbol{\tau}=\mathbf{3 0}$ & $\boldsymbol{\tau}=\mathbf{3 8}$ & $\boldsymbol{\tau}=\mathbf{7 7}$ \\
\hline 1 & 4.29 & 4.29 & 4.29 & 4.29 & 4.25 & 4.25 & 4.25 & 4.25 & 4.25 \\
2 & 3.65 & 3.65 & 3.65 & 3.65 & 3.60 & 2.35 & 3.08 & 3.65 & 3.65 \\
\hline
\end{tabular}




\begin{tabular}{llllllllll}
\hline \multirow{2}{*}{ Time } & \multicolumn{7}{l}{ Performance comparisons of optimization methods for different power price error coefficients } \\
\cline { 2 - 10 } & $\boldsymbol{\tau}=\mathbf{0}$ & $\boldsymbol{\tau}=\mathbf{1}$ & $\boldsymbol{\tau}=\mathbf{6}$ & $\boldsymbol{\tau}=\mathbf{1 0}$ & $\boldsymbol{\tau}=\mathbf{1 8}$ & $\boldsymbol{\tau}=\mathbf{2 5}$ & $\boldsymbol{\tau}=\mathbf{3 0}$ & $\boldsymbol{\tau}=\mathbf{3 8}$ & $\boldsymbol{\tau}=\mathbf{7 7}$ \\
\hline 3 & 5.03 & 5.03 & 5.03 & 5.03 & 4.14 & 4.58 & 5.03 & 5.03 & 5.03 \\
4 & 3.67 & 3.67 & 3.67 & 3.67 & 3.67 & 2.74 & 2.74 & 3.01 & 3.67 \\
5 & 3.65 & 3.65 & 4.15 & 4.15 & 4.15 & 4.12 & 4.12 & 3.62 & 3.62 \\
6 & 3.59 & 3.59 & 3.59 & 3.59 & 3.59 & 3.59 & 3.59 & 3.59 & 3.59 \\
7 & 2.21 & 2.21 & 2.71 & 2.71 & 2.71 & 2.71 & 2.44 & 2.10 & 2.21 \\
8 & 1.02 & 1.02 & 1.02 & 1.02 & 1.02 & 1.02 & 1.02 & 0.96 & 0.96 \\
9 & 2.61 & 2.61 & 2.61 & 2.61 & 2.61 & 2.61 & 2.55 & 2.55 & 2.55 \\
10 & 3.22 & 3.22 & 3.22 & 3.22 & 3.22 & 2.71 & 2.62 & 2.62 & 2.62 \\
12 & 6.27 & 6.27 & 4.27 & 4.27 & 3.62 & 3.62 & 3.62 & 5.62 & 5.62 \\
13 & 4.96 & 4.96 & 6.96 & 6.21 & 5.90 & 5.90 & 5.90 & 3.90 & 3.90 \\
14 & 6.43 & 6.43 & 4.43 & 4.43 & 4.17 & 4.17 & 4.17 & 6.17 & 6.17 \\
15 & 3.58 & 3.58 & 5.58 & 5.33 & 5.33 & 5.33 & 5.33 & 3.33 & 3.33 \\
16 & 6.79 & 6.79 & 4.79 & 6.59 & 6.59 & 6.48 & 6.35 & 7.35 & 7.35 \\
17 & 5.30 & 5.30 & 5.08 & 3.30 & 3.08 & 3.08 & 3.08 & 3.08 & 3.08 \\
18 & 4.04 & 4.04 & 3.42 & 3.42 & 3.28 & 3.28 & 3.28 & 3.28 & 3.28 \\
19 & 6.73 & 6.73 & 5.53 & 5.53 & 5.25 & 5.53 & 5.53 & 5.53 & 5.53 \\
20 & 5.85 & 5.85 & 5.82 & 5.82 & 5.82 & 5.82 & 5.82 & 5.82 & 5.82 \\
21 & 5.76 & 5.76 & 5.76 & 5.76 & 5.76 & 5.76 & 5.76 & 5.76 & 5.76 \\
22 & 6.07 & 6.07 & 6.07 & 6.07 & 6.07 & 6.07 & 6.07 & 6.07 & 6.07 \\
23 & 6.45 & 6.45 & 6.00 & 6.00 & 6.00 & 6.00 & 6.00 & 6.00 & 6.00 \\
24 & 3.98 & 3.98 & 2.98 & 2.94 & 2.44 & 2.44 & 2.44 & 2.44 & 2.44 \\
\hline
\end{tabular}

Table 4. Wind power declaration output in day-ahead market of virtual power plant with different scenarios of robustness.

\begin{tabular}{|c|c|c|c|c|c|c|c|c|c|}
\hline \multirow{2}{*}{ Time } & \multicolumn{9}{|c|}{ Wind power declaration output in day-ahead market of virtual power plant with different scenarios of robustness } \\
\hline & $\tau=\mathbf{0}$ & $\tau=1$ & $\tau=6$ & $\tau=\mathbf{1 0}$ & $\tau=\mathbf{1 8}$ & $\tau=\mathbf{2 5}$ & $\tau=\mathbf{3 0}$ & $\tau=\mathbf{3 8}$ & $\tau=77$ \\
\hline 1 & 4.50 & 4.50 & 4.50 & 4.50 & 4.46 & 4.46 & 4.46 & 4.46 & 4.46 \\
\hline 2 & 3.79 & 3.79 & 3.79 & 3.79 & 3.74 & 2.49 & 3.22 & 3.79 & 3.79 \\
\hline 3 & 5.08 & 5.08 & 5.08 & 5.08 & 4.19 & 4.63 & 5.08 & 5.08 & 5.08 \\
\hline 4 & 3.75 & 3.75 & 3.75 & 3.75 & 3.75 & 2.82 & 2.82 & 3.09 & 3.75 \\
\hline 5 & 3.77 & 3.77 & 4.27 & 4.27 & 4.27 & 4.24 & 4.24 & 3.74 & 3.74 \\
\hline 6 & 4.02 & 4.02 & 4.02 & 4.02 & 4.02 & 4.02 & 4.02 & 4.02 & 4.02 \\
\hline 7 & 3.12 & 3.12 & 3.62 & 3.62 & 3.62 & 3.62 & 3.35 & 3.01 & 3.12 \\
\hline 8 & 2.56 & 2.56 & 2.56 & 2.56 & 2.56 & 2.56 & 2.56 & 2.50 & 2.50 \\
\hline 9 & 0.85 & 0.85 & 0.85 & 0.85 & 0.85 & 0.85 & 0.79 & 0.79 & 0.79 \\
\hline 10 & 4.89 & 4.89 & 4.89 & 4.89 & 4.89 & 4.38 & 4.29 & 4.29 & 4.29 \\
\hline 12 & 3.25 & 3.25 & 1.25 & 1.25 & 0.60 & 0.60 & 0.60 & 2.60 & 2.60 \\
\hline 13 & 6.97 & 6.97 & 8.00 & 8.00 & 7.91 & 7.91 & 7.91 & 5.91 & 5.91 \\
\hline 14 & 7.67 & 7.67 & 5.67 & 5.67 & 5.41 & 5.41 & 5.41 & 7.41 & 7.41 \\
\hline 15 & 4.73 & 0.00 & 1.73 & 1.48 & 1.48 & 1.48 & 1.48 & 0.00 & 0.00 \\
\hline 16 & 2.74 & 7.74 & 5.74 & 7.54 & 7.54 & 7.43 & 7.30 & 8.00 & 8.00 \\
\hline 17 & 6.19 & 3.19 & 2.97 & 1.19 & 0.97 & 0.97 & 0.97 & 0.97 & 0.97 \\
\hline 18 & 4.05 & 5.25 & 4.63 & 4.63 & 4.49 & 4.49 & 4.49 & 4.49 & 4.49 \\
\hline 19 & 8.00 & 8.00 & 7.10 & 7.10 & 6.82 & 7.10 & 7.10 & 7.10 & 7.10 \\
\hline 20 & 8.00 & 8.00 & 7.97 & 7.97 & 7.97 & 7.97 & 7.97 & 7.97 & 7.97 \\
\hline 21 & 8.00 & 8.00 & 8.00 & 8.00 & 8.00 & 8.00 & 8.00 & 8.00 & 8.00 \\
\hline 22 & 8.00 & 8.00 & 8.00 & 8.00 & 8.00 & 8.00 & 8.00 & 8.00 & 8.00 \\
\hline 23 & 8.00 & 8.00 & 7.55 & 7.55 & 7.55 & 7.55 & 7.55 & 7.55 & 7.55 \\
\hline 24 & 4.92 & 4.92 & 3.92 & 3.88 & 3.38 & 3.38 & 3.38 & 3.38 & 3.38 \\
\hline
\end{tabular}


Table 5. Photovoltaic declaration output in day-ahead market of virtual power plant with different scenarios of robustness.

\begin{tabular}{|c|c|c|c|c|c|c|c|c|c|}
\hline \multirow{2}{*}{ Time } & \multicolumn{9}{|c|}{ Photovoltaic declaration output in day-ahead market of virtual power plant with different scenarios of robustness. } \\
\hline & $\tau=\mathbf{0}$ & $\tau=1$ & $\tau=6$ & $\tau=10$ & $\tau=18$ & $\tau=\mathbf{2 5}$ & $\tau=\mathbf{3 0}$ & $\tau=\mathbf{3 8}$ & $\tau=77$ \\
\hline 1 & 0.00 & 0.00 & 0.00 & 0.00 & 0.00 & 0.00 & 0.00 & 0.00 & 0.00 \\
\hline 2 & 0.00 & 0.00 & 0.00 & 0.00 & 0.00 & 0.00 & 0.00 & 0.00 & 0.00 \\
\hline 3 & 0.00 & 0.00 & 0.00 & 0.00 & 0.00 & 0.00 & 0.00 & 0.00 & 0.00 \\
\hline 4 & 0.00 & 0.00 & 0.00 & 0.00 & 0.00 & 0.00 & 0.00 & 0.00 & 0.00 \\
\hline 5 & 0.00 & 0.00 & 0.00 & 0.00 & 0.00 & 0.00 & 0.00 & 0.00 & 0.00 \\
\hline 6 & 0.00 & 0.00 & 0.00 & 0.00 & 0.00 & 0.00 & 0.00 & 0.00 & 0.00 \\
\hline 7 & 0.00 & 0.00 & 0.00 & 0.00 & 0.00 & 0.00 & 0.00 & 0.00 & 0.00 \\
\hline 8 & 0.00 & 0.00 & 0.00 & 0.00 & 0.00 & 0.00 & 0.00 & 0.00 & 0.00 \\
\hline 9 & 3.00 & 3.00 & 3.00 & 3.00 & 3.00 & 3.00 & 3.00 & 3.00 & 3.00 \\
\hline 10 & 0.00 & 0.00 & 0.00 & 0.00 & 0.00 & 0.00 & 0.00 & 0.00 & 0.00 \\
\hline 12 & 5.00 & 5.00 & 5.00 & 5.00 & 5.00 & 5.00 & 5.00 & 5.00 & 5.00 \\
\hline 13 & 0.00 & 0.00 & 0.97 & 0.22 & 0.00 & 0.00 & 0.00 & 0.00 & 0.00 \\
\hline 14 & 0.00 & 0.00 & 0.00 & 0.00 & 0.00 & 0.00 & 0.00 & 0.00 & 0.00 \\
\hline 15 & 0.00 & 4.73 & 5.00 & 5.00 & 5.00 & 5.00 & 5.00 & 4.48 & 4.48 \\
\hline 16 & 5.00 & 0.00 & 0.00 & 0.00 & 0.00 & 0.00 & 0.00 & 0.30 & 0.30 \\
\hline 17 & 0.00 & 3.00 & 3.00 & 3.00 & 3.00 & 3.00 & 3.00 & 3.00 & 3.00 \\
\hline 18 & 1.20 & 0.00 & 0.00 & 0.00 & 0.00 & 0.00 & 0.00 & 0.00 & 0.00 \\
\hline 19 & 0.30 & 0.30 & 0.00 & 0.00 & 0.00 & 0.00 & 0.00 & 0.00 & 0.00 \\
\hline 20 & 0.00 & 0.00 & 0.00 & 0.00 & 0.00 & 0.00 & 0.00 & 0.00 & 0.00 \\
\hline 21 & 0.00 & 0.00 & 0.00 & 0.00 & 0.00 & 0.00 & 0.00 & 0.00 & 0.00 \\
\hline 22 & 0.00 & 0.00 & 0.00 & 0.00 & 0.00 & 0.00 & 0.00 & 0.00 & 0.00 \\
\hline 23 & 0.00 & 0.00 & 0.00 & 0.00 & 0.00 & 0.00 & 0.00 & 0.00 & 0.00 \\
\hline 24 & 0.00 & 0.00 & 0.00 & 0.00 & 0.00 & 0.00 & 0.00 & 0.00 & 0.00 \\
\hline
\end{tabular}

Table 6. Profit curves with different robustness coefficients and deviation penalty coefficients.

\begin{tabular}{lccccc}
\hline \multirow{\tau}{*}{} & \multicolumn{5}{l}{ Profit curves with different robustness coefficients and deviation penalty coefficients } \\
\cline { 2 - 6 } & $\boldsymbol{\theta}=\mathbf{0 . 3 6}$ & $\boldsymbol{\theta}=\mathbf{0 . 3 8}$ & $\boldsymbol{\theta}=\mathbf{0 . 4}$ & $\boldsymbol{\theta}=\mathbf{0 . 4 2}$ & $\boldsymbol{\theta}=\mathbf{0 . 4 4}$ \\
\hline 0 & 34170.28 & 34334.61 & 34681.95 & 35087.71 & 35552.76 \\
1 & 33063.62 & 33228.33 & 33574.30 & 33980.05 & 34445.11 \\
6 & 29401.15 & 29569.23 & 29737.02 & 30027.55 & 30402.67 \\
10 & 28401.78 & 28565.08 & 28728.33 & 28891.24 & 29060.64 \\
18 & 27212.27 & 27375.82 & 27541.52 & 27710.78 & 27882.66 \\
25 & 26719.29 & 26884.10 & 27050.42 & 27220.94 & 27396.57 \\
30 & 26518.57 & 26674.26 & 26831.74 & 26991.60 & 27163.52 \\
38 & 26364.77 & 26512.85 & 26661.58 & 26810.75 & 26967.48 \\
77 & 26318.49 & 26463.54 & 26608.59 & 26754.66 & 26907.57 \\
\hline
\end{tabular}

\section{Conclusion}

The model is validated by the example data. The results show that the model proposed in this paper can obtain the most robust day-ahead declaration decision-making plan by introducing robust method based on the uncertainty of the day-ahead and real-time market electricity price and the distributed energy output. By changing the robust uncertainty coefficient, the conservativeness of the virtual power plant optimization scheme can be flexibly adjusted, which is beneficial for decision makers of virtual power plant to make a reasonable choice between probability of operating revenue and violation of constraint risk. The advantage of the optimization method over the deterministic optimization is enhanced with the increase of the prediction error, and the obtained day-ahead declared output scheme has strong robustness and the ability to withstand the risk of market electricity price fluctuations, which verifies the validity of the model constructed in this paper.

\section{References}

[1] Jie Xiao, Xiangyu Kong, Qiang Jin, Hengxu You, Kai Cui, Yusen Zhang, Demand-Responsive Virtual Power Plant Optimization Scheduling Method Based on Competitive Bidding Equilibrium, Energy Procedia, Volume 152,2018, Pages 1158-1163, ISSN 1876-6102. 
[2] Yangyang Liu, Min Li, Hongbo Lian, Xiaowei Tang, Chuanquan Liu, Chuanwen Jiang, Optimal dispatch of virtual power plant using interval and deterministic combined optimization, International Journal of Electrical Power \& Energy Systems, Volume 102, 2018, Pages 235-244, ISSN 0142-0615.

[3] Congying Wei, Jian Xu, Siyang Liao, Yuanzhang Sun, Yibo Jiang, Deping Ke, Zhen Zhang, Jing Wang, A bi-level scheduling model for virtual power plants with aggregated thermostatically controlled loads and renewable energy, Applied Energy, Volume 224, 2018, Pages 659-670, ISSN 0306-2619.

[4] Mahmoud M. Othman, Y. G. Hegazy, Almoataz Y. Abdelaziz, Electrical energy management in unbalanced distribution networks using virtual power plant concept, Electric Power Systems Research, Volume 145, 2017, Pages 157-165, ISSN 0378-7796.

[5] Pandžić H, Morales J M, Conejo A J, et al. Offering model for a virtual power plant based on stochastic programming $[\mathrm{J}]$. Applied Energy, 2013, 105(5):282-292.

[6] Morteza R, Luis B. Strategic Bidding for a Virtual Power Plant in the Day-Ahead and Real-Time Markets: A Price-Taker Robust Optimization Approach [J]. IEEE Transactions on Power Systems, 2016, 31(4):2676-2687.

[7] Mashhour E, Moghaddas-Tafreshi S M. Bidding Strategy of Virtual Power Plant for Participating in Energy and Spinning Reserve Markets-Part II: Numerical Analysis [J]. IEEE Transactions on Power Systems, 2011, 26(2):957-964.
[8] Dantzig, G. B. (1955). Linear programming with uncertainty. Management Science, 1,197-206.

[9] Wei Wei, Liu Feng, Shengwei Mei. Power System Robust Economic Dispatch (1) Theoretical basis [J]. Automation of Electric Power Systems, 2013, 37(17):37-43.

[10] Ying Chen, Zhihong Yuan, Bingzhen Chen, Process optimization with consideration of uncertainties-An overview, Chinese Journal of Chemical Engineering, Volume 26, Issue 8, 2018.

[11] Dupacova, J., Growe-Kuska, N., \& Romisch, W. (2003). Scenario reduction in stochastic programming: An approach using probability metrics. Mathematical Programming, Ser. A 95, 493511. doi: 10.1007/s10107-002-0331-0.

[12] Qianwen Zhang, Xiuli Wang, Tianyan Yang, etc. Robust Optimal Scheduling for Power Systems with Wind Farms [J]. Power grid technology, 2017, 41(5):102-114.

[13] Jiang R, Wang J, Guan Y. Robust Unit Commitment with Wind Power and Pumped Storage Hydro [J]. IEEE Transactions on Power Systems, 2012, 27(2):800-810.

[14] Dantzig G B, Thapa M N. Linear programming 2: theory and extensions [M]. Springer Science \& Business Media, 2006.

[15] Valentin Robu, Georgios Chalkiadakis, Ramachandra Kota, Alex Rogers, Nicholas R. Jennings, Rewarding cooperative virtual power plant formation using scoring rules, Energy, Volume 117, Part 1, 2016, Pages 19-28, ISSN 0360-5442. 